\title{
NeuroD Modulates Opioid Agonist-Selective Regulation of Adult Neurogenesis and Contextual Memory Extinction
}

\author{
Hui Zheng, ${ }^{* 1,2}$, Yue Zhang ${ }^{2}$, Wen Li', Horace H Loh' ${ }^{2}$ and Ping-Yee Law ${ }^{2}$ \\ 'Key Laboratory of Regenerative Biology of CAS, Guangdong Provincial Key Laboratory of Stem Cell and Regenerative Medicine, South China \\ Institute for Stem Cell Biology and Regenerative Medicine, Guangzhou Institutes of Biomedicine and Health, Chinese Academy of Sciences, \\ Science City, Guangzhou, China; ${ }^{2}$ Department of Pharmacology, University of Minnesota, Minneapolis, MN, USA
}

\begin{abstract}
Addictive drugs, including opioids, modulate adult neurogenesis. In order to delineate the probable implications of neurogenesis on contextual memory associated with addiction, we investigated opioid agonist-selective regulation of neurogenic differentiation I (NeuroD) activities under the conditioned place preference (CPP) paradigm. Training mice with equivalent doses of morphine and fentanyl produced different CPP extinction rates without measurable differences in the CPP acquisition rate or magnitude. Fentanylinduced CPP required much longer time for extinction than morphine-induced CPP. We observed a parallel decrease in NeuroD activities and neurogenesis after morphine-induced CPP, but not after fentanyl-induced CPP. Increasing NeuroD activities with NeuroDlentivirus (nd-vir) injection at the dentate gyrus before CPP training reversed morphine-induced decreases in NeuroD activities and neurogenesis, and prolonged the time required for extinction of morphine-induced CPP. On the other hand, decreasing NeuroD activities via injection of miRNA-190-virus (190-vir) reversed the fentanyl effect on NeuroD and neurogenesis and shortened the time required for extinction of fentanyl-induced CPP. Another contextual memory task, the Morris Water Maze (MWM), was affected similarly by alteration of NeuroD activities. The reduction in NeuroD activities either by morphine treatment or 190-vir injection decreased MWM task retention, while the increase in NeuroD activities by nd-vir prolonged MWM task retention. Thus, by controlling NeuroD activities, opioid agonists differentially regulate adult neurogenesis and subsequent contextual memory retention. Such drugrelated memory regulation could have implications in eventual context-associated relapse.
\end{abstract}

Neuropsychopharmacology (2013) 38, 770-777; doi:I 0.1038/npp.20 I2.242; published online I 6 January 2013

Keywords: agonist-selective signaling; NeuroD activity; adult neurogenesis; contextual memory

\section{INTRODUCTION}

Addictive drugs, acting as reinforcers, override the controls of the neural circuitries that are normally involved in pleasure, incentive motivation, and learning (Chao and Nestler, 2004; Robinson and Berridge, 2003). It is apparent that both learning and affective dysregulation are the driving forces in addictive behaviors, intense cravings for drugs, and relapse to drug-using behavior. In addition to the probable involvement of corticotropin-releasing factor and the dopaminergic system within the nucleus accumbens and ventral tegmental area, glutaminergic inputs from the prefrontal cortex, amygdala and hippocampus are also involved in the reinstatement of drug-seeking behavior in animals (Aguilar et al, 2009). In particular, the hippocampus has a substantial role in drug acquisition and relapse, as well as in the drug reward experience, as supported by the

*Correspondence: Dr H Zheng, Guangzhou Institutes of Biomedicine and Health, A-131, 190 Kaiyuan Ave., Science City, Guangzhou 510530, China, Tel: + 86203201 5334, Fax: + 86203201 5231, E-mail: zheng_hui@gibh.ac.cn

Received 7 March 2012; revised 12 November 2012; accepted 13 November 2012; accepted article preview online 29 November 2012 following studies: (1) injection of inhibitors into the hippocampus disrupted reactivation of morphine-induced memories (Milekic et al, 2006); (2) injection of CaMKII inhibitors into dentate gyrus (DG) blocked morphineinduced conditioned place preference (CPP) response (Fan et al, 1999); and (3) functional inactivation of dorsal hippocampus with tetrodotoxin abolished context-induced reinstatement in rats previously trained in self-administration (Fuchs et al, 2005). Recent studies indicate that the hippocampus leads the ventral striatum in the replay of place reward information (Lansink et al, 2009), and that hippocampus CA3 principal cells are more active after receiving a reward (Singer and Frank, 2009). These data and others solidify the roles of the hippocampus and contextual memory in the association between drug experiences and reward. Disruption of this memory reconsolidation could abolish the association between context and drugs, which could lead to relapse (Lee, 2008).

The differentiation of progenitor cells or neural stem cells within the subgranular zone (SGZ) of the DG into newborn granular neurons contributes to information processing in the adult hippocampus (Abrous et al, 2005). Addictive drugs such as morphine, nicotine, ethanol, and cocaine are able to regulate adult neurogenesis in the SGZ (Eisch and 
Harburg, 2006). Although many factors can affect adult neurogenesis (see review by Zhao et al, 2008), the exact mechanism by which addictive drugs regulate neurogenesis remains unknown.

Morphine and fentanyl, two commonly prescribed opioid analgesic drugs, are highly addictive. Although they are both $\mu$-opioid receptor (OPRM1) agonists, morphine activates ERK via the PKC pathway while fentanyl uses the $\beta$-arrestin-dependent pathway (Zheng et al, 2008). Such pathway selectivity leads to their differential regulation of expression of miRNA-190 (miR-190) and one of its targets, neurogenic differentiation 1 (NeuroD) (Zheng et al, 2010b). As both NeuroD and one of its targets, doublecortin (DCX) (Seo et al, 2007), are important factors in adult neurogenesis in the SGZ (Couillard-Despres et al, 2005; von Bohlen Und Halbach, 2007), differential control of NeuroD activities by these two agonists could manifest as differential abilities of the hippocampus to store, consolidate, or retrieve memory. To test this hypothesis, we examined the effects of NeuroD activities on two behavioral responses: (1) the drug-induced CPP response, which has been used to demonstrate that re-exposure to a drug or stressful event can induce reacquisition or reinstatement of drug behavior in animals (Tzschentke, 2007); and (2) the Morris Water Maze (MWM), which is used to monitor the spatial learning of rodents (Vorhees, 2006).

\section{MATERIALS AND METHODS}

\section{Animals}

Six to eight-week-old CD-1 (ICR) male mice from Charles River (Portage, MI) were housed in groups of four with access to food and water ad libitum. All procedures were taken in accordance with IACUC policies. Mice were allowed to habituate for 1 week before experiments.

\section{Virus Injection}

Lentiviruses (containing a CMV promoter) expressing miR190 (190-vir), NeuroD-lentivirus (nd-vir), or a control oligonucleotide (Con-vir) was prepared as described previously (Zheng et al, 2010a). Mice were anesthetized with $90 \mathrm{mg} / \mathrm{kg}$ ketamine and $10 \mathrm{mg} / \mathrm{kg}$ xylazine i.p. A burr hole of $0.5 \mathrm{~mm}$ was drilled $-2.1 \mathrm{~mm}$ posterior to the bregma and $\pm 1.1 \mathrm{~mm}$ lateral to the midline. A $10-\mu \mathrm{l}$ syringe filled with virus was lowered $1.7 \mathrm{~mm}$ below the meniscus in a 2-min period (Cetin et al, 2006). Using a micro-syringe pump controller, $1 \mu \mathrm{l}$ of virus $\left(6 \times 10^{8} \mathrm{TU} / \mathrm{ml}\right)$ was injected at a constant rate over a 10 -min period. The virus was allowed to diffuse for $5 \mathrm{~min}$ and the needle was then raised slowly at a constant rate over a 2 -min period. The holes were sealed with bone wax and the mice were allowed to recover for 1 week before behavioral tests.

\section{Conditioned place preference}

CPP was carried out in a three chamber apparatus supplied by Panlab (Harvard Apparatus, Holliston, MA) according to the protocol (Cunningham et al, 2006). In the habituation section, mice were placed in the test room $2 \mathrm{~h}$ before any handling. The mice were then allowed free access to the whole CPP apparatus for 30 min per day for 2 days. In the preconditioning section (day 1-9), place preference was tested on day 1 by placing each mouse individually into the CPP apparatus at the neutral middle chamber, with free access to the two side chambers for $10 \mathrm{~min}$. Mice with no significant preference for the two chambers on day 1 were divided randomly for the stereotaxic lentivirus injection. Mice were allowed to recover from day 2-8, and subjected to another place preference test on day 9. In the conditioning section (day 10-14), half of the mice received either $5 \mathrm{mg} / \mathrm{kg}$ morphine or $50 \mu \mathrm{g} / \mathrm{kg}$ fentanyl subcutaneously in the morning and an equal volume of saline subcutaneously in the afternoon. In order to eliminate the circadian rhythm effects on opiate responses (Weed, 2006), the other half of the mice received a reversed injection paradigm, ie, saline in the morning and drug in the afternoon. The injection paradigm and drug-paired compartment were selected randomly. In the post-conditioning section, the place preference of the mice was tested once a week until no significant preference was detected in two continuous tests. CPP time was calculated with the following equation: (time in drug-paired chamber)-(time in saline-paired chamber). In the reinstatement section, mice were injected with $5 \mathrm{mg} / \mathrm{kg}$ morphine or $50 \mu \mathrm{g} / \mathrm{kg}$ fentanyl 1 week after the last test in the previous section. Mice were then placed in the CPP apparatus for recording of place preference. AnyMaze software (Stoelting, Wood Dale, IL) was used to track the mice.

\section{Morris Water Maze}

MWM was performed as reported (Vorhees, 2006). In the acquisition section, the mice were placed in the water maze $(122 \mathrm{~cm}$ diameter) with the platform $(10 \mathrm{~cm}$ diameter) submerged in the SW quadrant. The mice were trained for four trails per day for 5 days with a semi-random set of start positions as previously published (Vorhees, 2006). AnyMaze software was used to track the mice. In the extinction section, mice were placed in the NE quadrant of the water maze without the platform for $60 \mathrm{~s}$. The amount of time the mice spent in each quadrant searching for the platform was recorded. Probe tests were performed every 4 days afterward until the mice spent an equal amount of time in each quadrant.

\section{Histology and Immunohistochemistry}

Mice were deeply anesthetized with ketamine and xylazine and perfused with $4 \%$ paraformaldehyde. Brains were postfixed by transferring to $4 \%$ paraformaldehyde, $10 \%$ sucrose, $20 \%$ sucrose, and $30 \%$ sucrose sequentially for $24 \mathrm{~h}$ each at $4{ }^{\circ} \mathrm{C}$. Twenty micrometer coronal tissue sections were prepared and mounted on Superfrost Plus glass slides (Thermo Fisher Scientific, Waltham, MA). Every seventh section across the hippocampus was stained with either NeuroD antibodies (Cell Signaling, Danvers, MA) or DCX antibodies (Santa Cruz Biotechnology, Santa Cruz, CA) and immunohistochemically analyzed as described (Hodge et al, 2008). A BD CARV II Confocal Imager connected to a Leica DMIRE2 fluorescence microscope with a Hamamatsu EMCCD C9100 camera and the software Metamorph (Molecular 
Devices, Sunnyvale, CA) were used for data acquisition and analyses.

\section{RESULTS AND DISCUSSION}

The agonist-selective regulation of NeuroD activities and dendritic spine morphology has been observed in primary hippocampus neurons (Zheng et al, 2010a). As dendritic spines contribute to the formation of contextual memory (Restivo et al, 2009), and as the hippocampus and contextual memory have been linked to drug acquisition and relapse (Fan et al, 1999; Milekic et al, 2006), we surmised that CPP response, a hippocampus-mediated contextual memory behavior test that measures the reacquisition or reinstatement of drug experience in rodents (Shaham et al, 2003; Tzschentke, 2007), should exhibit agonist-selective response. Hence, CD-1 mice were tested with the CPP acquisition and extinction paradigms as outlined in Supplementary Figure S1. As shown in Supplementary Figure S2A and S2B, our current CPP paradigm produced morphine and fentanyl dose-dependent responses. Morphine $(5 \mathrm{mg} / \mathrm{kg})$ and fentanyl $(50 \mu \mathrm{g} / \mathrm{kg})$ were chosen for subsequent CPP studies because these doses allowed us to examine whether NeuroD activities could regulate the magnitude of the CPP response. After five-conditioning sessions, mice trained with morphine or fentanyl had a similar preference for the drug-paired chamber (Figure 1). However, there was a dramatic difference in extinction rates between the mice trained with morphine and the mice trained with fentanyl. Morphine-induced CPP returned to basal levels at day 30 while fentanyl-induced CPP returned to basal levels at day 79 (Figure 1).

The difference in extinction rates may be due to the distinct abilities to form new place preference memory or 'inhibitory memory', as the test for extinction is similar to the training that both compartments are paired with no drug injection. To exclude this possibility, place preference was measured at a lower frequency (only on day 15, day 44, and day 79) (Supplementary Figure S2C), and the differences in extinction rates persisted. The difference in extinction rates also could have arisen from the difference in the number of conditioning sections needed by the two agonists to acquire similar CPP response. As summarized in Supplementary Figure S2D, equivalent doses of morphine and fentanyl resulted in similar sessions for maximal CPP response.

The antinociceptive effects of morphine and fentanyl peaked at 30 and $15 \mathrm{~min}$, respectively (Zheng et al, 2010b). However, reducing the time of CPP training with fentanyl to $15 \mathrm{~min}$ or increasing the time of CPP training with morphine to $60 \mathrm{~min}$ did not alter CPP acquisition or extinction (Supplementary Figure S2E and S2F), suggesting that the difference in pharmacokinetics was not essential under the current paradigm. In addition, administering the drugs either in the morning or in the afternoon did not alter the CPP responses (Supplementary Figure S2G and S2H). Hence, the differences in CPP extinction were not consequences of conditioned place aversion due to drug withdrawal (Rothwell et al, 2010).

As adult neurogenesis in the DG has been shown to be: (1) critical in hippocampus-dependent complex pattern

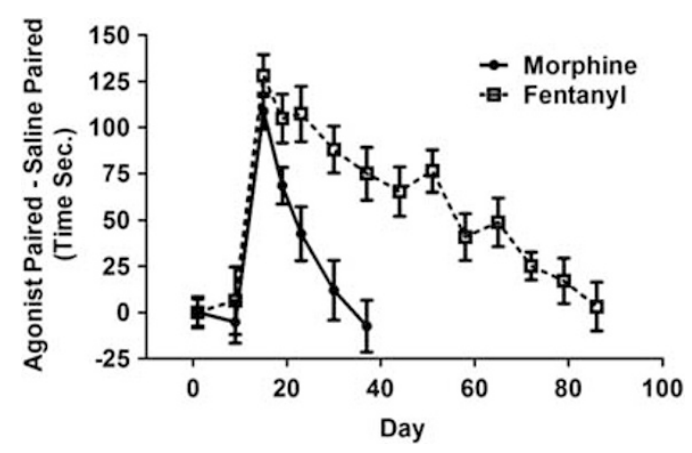

Figure I The extinction of fentanyl-induced conditioned place preference (CPP) is slower than that of morphine-induced CPP. Mice not receiving lentivirus injection on day I were used for CPP training (from day 10 to day 14) with morphine or fentanyl. Place preference was tested every 7 days until no significant place preference was detected in two continuous tests. Error bars represent SE $(n=10)$.

recognition (Clelland et al, 2009); (2) affected by environment and experience (Brown et al, 2003); and (3) dependent on the morphine treatment paradigm (Fischer et al, 2008), we examined whether the current CPP paradigm would affect adult neurogenesis by monitoring the transcript and protein levels of DCX in the hippocampus (CouillardDespres et al, 2005). As shown in Figure 2, transcript and protein levels of DCX were reduced in morphine-trained mice. These decreases were consistent with a reduction in NeuroD activity, as indicated by phosphorylated NeuroD (pNeuroD) levels; this was possibly due to impaired CaMKII $\alpha$ activity (Figure $2 b$ ). On the other hand, NeuroD transcript levels increased in fentanyl-trained mice due to a decrease in miR-190 levels (Figure 2a). An increase in NeuroD protein levels led to no alteration in pNeuroD levels, because fentanyl also suppressed CaMKII $\alpha$ activity (Figure 2b). Subsequently, DCX transcript and protein levels were not affected in the fentanyl-trained mice (Figure 2). These observations are in accord with our reported studies in primary hippocampus neuron cultures (Zheng et al, 2010a) and suggest that adult neurogenesis is differentially regulated by morphine and fentanyl in vivo in the CPP paradigm.

The immunohistofluorescence analyses of NeuroD + and DCX + cells in the DG revealed results consistent with immunoblotting of NeuroD and DCX, respectively (Figure 3 and Supplementary Figure S3). The number of NeuroD + cells is not indicative of NeuroD activity because CaMKII $\alpha$ mediated phosphorylation is required for its activation (Gaudilliere et al, 2004). DCX is one of NeuroD's targets (Seo et al, 2007), thus DCX levels are indicative not only of the later stage of neural differentiation during adult neurogenesis but also of NeuroD activity. In mice conditioned with morphine, the number of NeuroD + cells was unchanged, but the number of DCX + cells decreased by $61 \pm 6 \%$. In contrast, in mice conditioned with fentanyl, the number of NeuroD + cells increased by $119 \pm 33 \%$ while the number of DCX + cells was unchanged (Figure 3). To clarify the effects of various treatments and the factors mentioned above, the results shown in Figures 2-4 are summarized in Supplementary Table S1.

In hippocampus, the highest NeuroD mRNA level could be observed in SGZ, but the NeuroD mRNA could also be 


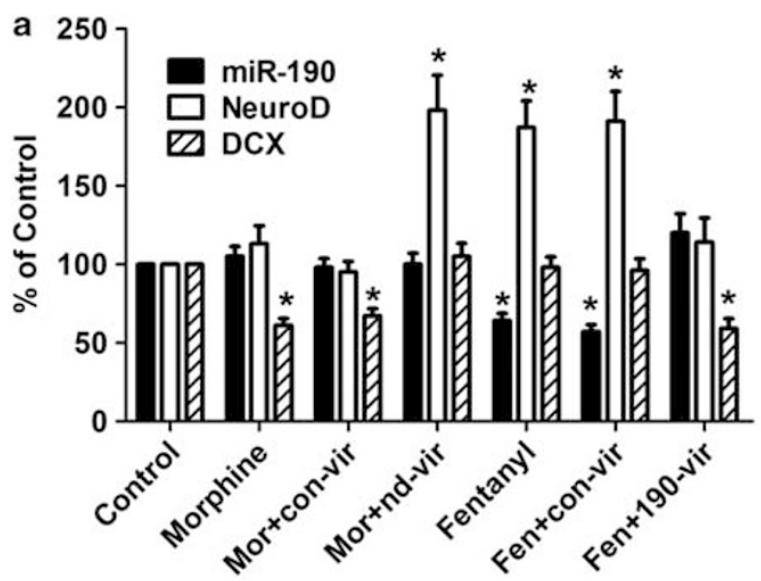

b $\quad$ p NeuroD
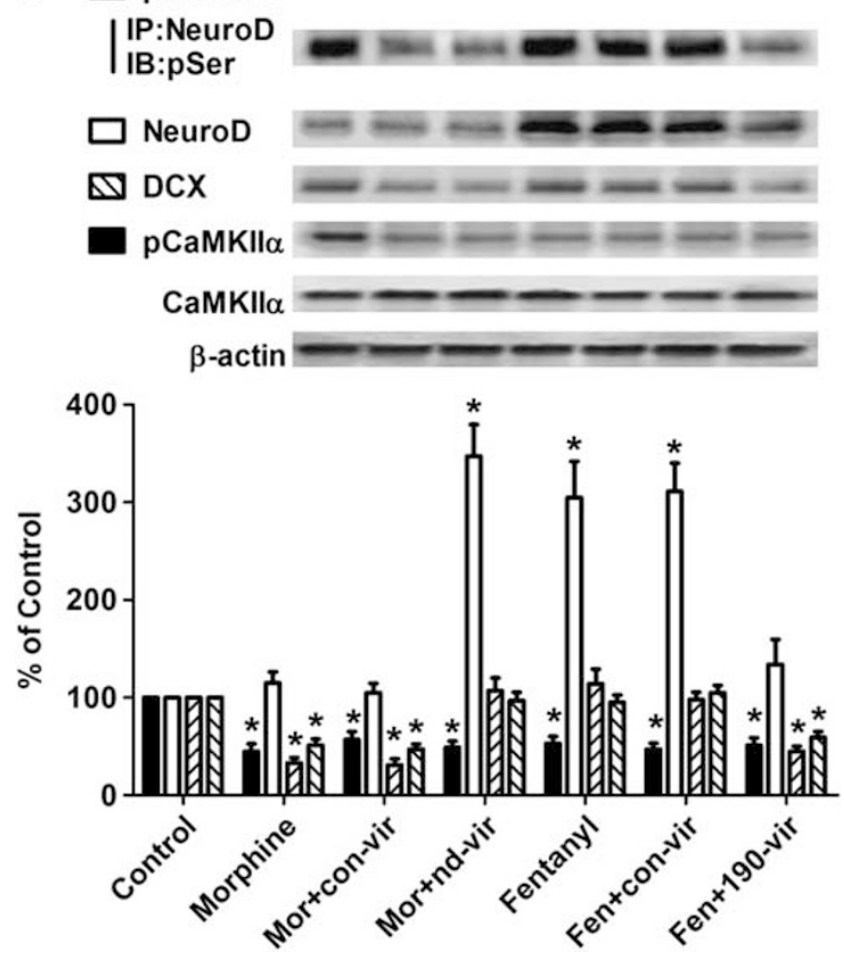

Figure 2 Neurogenic differentiation I (NeuroD) activity on day 15. Control mice received no virus injection or conditioned place preference (CPP) training. The other six groups of mice were injected with vehicle, control oligonucleotide (con-vir), NeuroD-lentivirus (nd-vir), or 190-virus (190-vir) on day I and then received CPP training with morphine or fentanyl from day 10 to day 14 as in Supplementary Figure SI. Mouse dentate gyrus (DG) were used on day 15. Mor and Fen represent morphine and fentanyl, respectively. (a) The expression levels of miR-190, NeuroD, and DCX were measured with qRT-PCR. GAPDH was used as internal control for normalization. (b) The amounts of NeuroD, phosphorylated NeuroD (pNeuroD, phosphorylated serine (pSer) in precipitated NeuroD), CaMKIl $\alpha$, phosphorylated CaMKIl $\alpha$ (pCaMKIl $\alpha$ ), and DCX were determined with immunoblotting and normalized against $\beta$ actin. One-way ANOVA with Dunnett's test as a post-hoc test was used for analysis. Comparisons were done between the 'Control' and other groups. Error bars and '*' represent SD $(n=4)$ and $P<0.0$ I, respectively.

observed in other place of as demonstrated in Allen Brain Atlas (http://www.brain-map.org, experiment no. 75650865 and 79632311), which is consistent with previous reports (Lee et al, 2000; McCormick et al, 1996; Uittenbogaard and Chiaramello, 2000). However, NeuroD protein was restricted to progenitors/neuroblasts (type $2 \mathrm{~b}$ and 3 cells) located in the SGZ. This could be due to post-transcriptional regulation, such as miR-mediated control of transcript levels. For example, in addition to miR-190, miR-19b and miR-124 also regulate NeuroD expression (Liu et al, 2010; Zhang et al, 2011). The existence of post-transcriptional regulation is also supported by studies on exogenously expressed NeuroD in fetal astroglial cells and zebrafish (Kamath et al, 2005; Thomas et al, 2012), which explains why nd-vir injection did not produce a widespread expression of NeuroD in the DG. Such restricted expression of NeuroD could be demonstrated further by the expression of NeuroD 2 days post injection in all cells adjacent to the injection sites, but restricted to few cells 7 days post injection (Supplementary Figure S4). As Supplementary Figure S4 was prepared on day 2 and day 7 after virus injection, similar injection tissue damage was not noticeable in Figure 3 and Supplementary Figure S3, which were collected on day 15 after virus injection. Thus we had a seven-day rest for mice before the CPP training (Supplementary Figure S1).

If regulation of neurogenesis is related to the observed difference in morphine- $v s$ fentanyl-induced CPP extinction rates, then modulation of NeuroD activity should affect these rates. We demonstrated, using qRT-PCR, western blot, and immunohistofluorescence analyses (and Supplementary Figure S3), that injection of either nd-vir or 190-vir increased or decreased NeuroD activity and neurogenesis, respectively. Interestingly, parallel to endogenous NeuroD expression, injection of nd-vir into the DG increased the number of NeuroD + cells at the SGZ (Figure 3 and Supplementary Figure S3), probably due to post-transcriptional regulation as discussed above. Nevertheless, when CPP extinction rates were measured, nd-vir significantly increased the extinction time of the morphineinduced CPP response: extinction time was 65 days vs 30 days in mice injected with con-vir or no virus (Figure 4a). On the other hand, attenuating NeuroD activity with 190-vir injection dramatically accelerated the extinction of fentanylinduced CPP to 44 days instead of 79 days in mice injected with con-vir or no virus (Figure $4 \mathrm{~b}$ ).

The reinstatement of extinguished CPP responses after a non-contingent administration of a priming dose of drug has been accepted as reliable measurement for drug craving and relapse (Shalev et al, 2002; Zheng et al, 1999). Thus, CPP reinstatement was tested 1 week after total extinction by challenging the mice with the same dose of morphine or fentanyl used in the conditioning section. As summarized in Figure $4 \mathrm{c}$, the magnitude of reinstatement of morphineinduced CPP was much lower than that of fentanyl-induced CPP. Injecting nd-vir but not con-vir increased the magnitude of reinstatement in morphine-trained mice, whereas injecting 190-vir decreased the magnitude of reinstatement in fentanyl-trained mice (Figure 4c). Therefore, by regulating NeuroD activity, both the extinction and the reinstatement, but not the acquisition, of CPP response can be modulated.

We examined whether NeuroD activity can modulate overall contextual memory using another memory task, MWM, as outlined in Supplementary Figure S5. As indicated in Supplementary Figure S6, injection with 

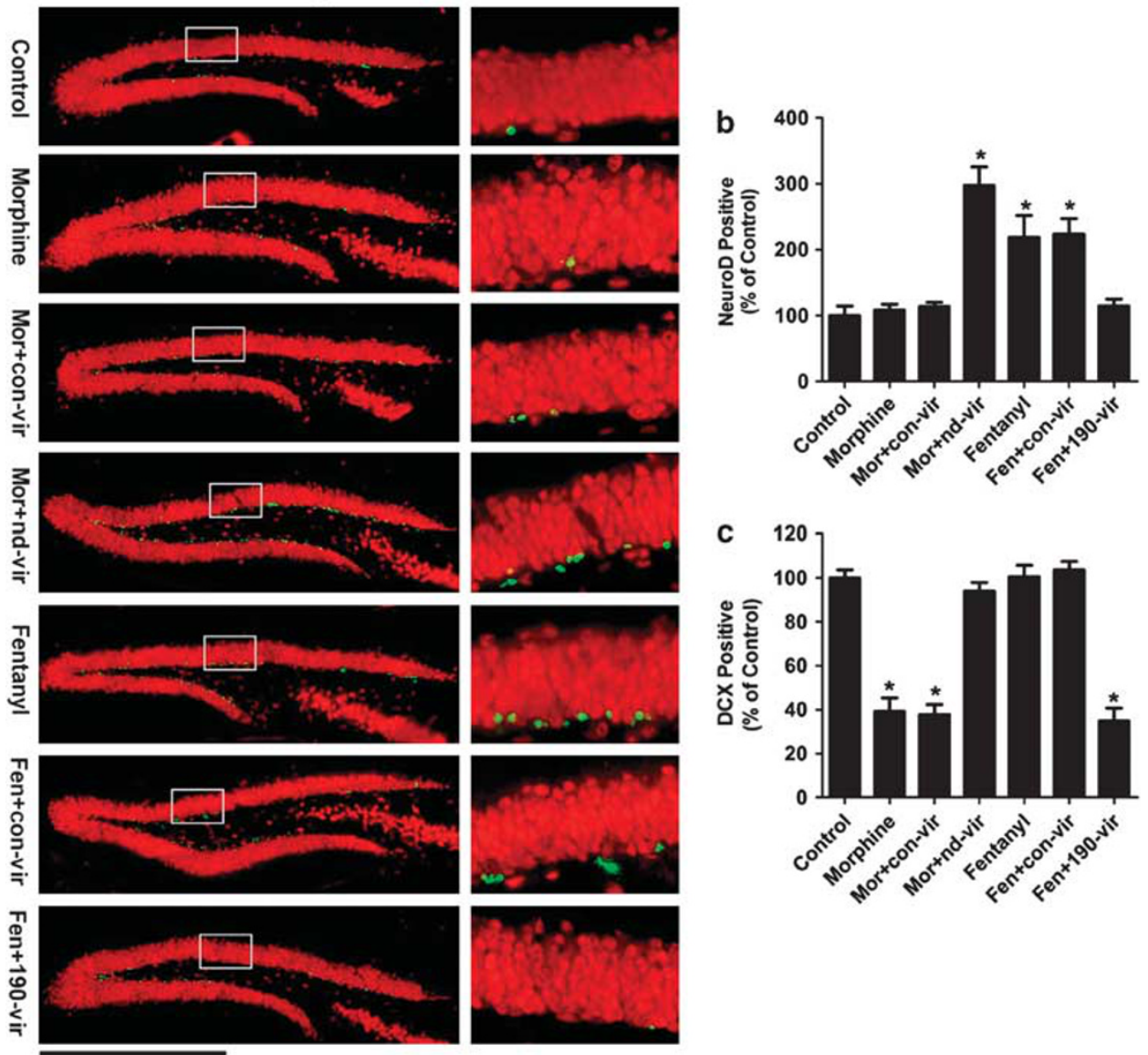

Figure 3 Neurogenic differentiation I (NeuroD) activity regulates adult neurogenesis. Seven groups of mice were trained as in Figure 2. Slides of dentate gyrus (DG) were prepared on day 15. (a) The staining of NeuroD + cells in the seven groups. Bar equals $500 \mu$ M. (b and c) A summary of NeuroD + (b) and DCX + (c) cell numbers across the whole DG, normalized to control mice. One-way ANOVA with Dunnett's test as a post-hoc test was used for analysis. Comparisons were done between the 'Control' and other groups. Error bars and '*' represent SD $(n=10)$ and $P<0.01$, respectively.

190-vir or nd-vir alone (without any agonist treatment) led to consistent changes in NeuroD protein levels, the amount of pNeuroD, and DCX expression, as there is no agonist to alter CaMKII $\alpha$ activity. Although there was a training session-dependence in recognition of the submerged platform's location (SW), injection of nd-vir or 190-vir to the DG did not alter the rate at which the mice learned the platform's location (Figure 5a). However, manipulation of NeuroD activity dramatically altered the rate at which the mice forgot the platform's location. As shown in Figure 5b, the mice in the control group spent more time in the SW quadrant when probed the next day with the platform removed. Mice injected with 190-virus forgot the platform location 12 days after the initial probe, while mice injected with con-vir, nd-vir, or 190-vir + nd-vir remembered the platform's location for up to 24 days (Figure 5b).

We also examined whether morphine or fentanyl could differentially regulate this NeuroD-dependent contextual memory task. After lentivirus or vehicle injection, subcutaneous injection of $5 \mathrm{mg} / \mathrm{kg}$ morphine or $50 \mu \mathrm{g} / \mathrm{kg}$ fentanyl per day for 5 days (a similar paradigm to the the $\mathrm{CPP}$-conditioning section but without CPP training) did not alter the acquisition rate (Supplementary Figure S7). Interestingly, mice injected with morphine showed a dramatic decrease in the time they remembered the platform's location, which could be restored by the preinjection of nd-vir (Figure 5c). On the other hand, mice injected with fentanyl before MWM training forgot the platform's location at a similar rate as the saline injected mice (Figure 5d). Again, reducing NeuroD activity with 190vir injection increased the rate at which the fentanylinjected mice forgot the platform's location but exhibited no effect on their learning of the platform's location (Figure $5 \mathrm{~d}$ and Supplementary Figure S7J). Thus, as in CPP trials, NeuroD activity affected the retention but not the acquisition of contextual memory in the MWM test.

From both contextual memory tasks, it is apparent that the opioid regulation of adult neurogenesis in the DG is dependent on NeuroD activity. A schematic illustration of the current study is provided in Supplementary Figure S8. The integrity of the DG in regulating contextual memory related to drug response is well established. Disruption of the DG results in a slower acquisition rate and a deficit in long-term contextual retrieval of fear memory (Lee and Kesner, 2004). Neurotoxic lesions that damage the DG prevent the acquisition of both cocaine-induced CPP and context-conditioned fear response (Hernandez-Rabaza et al, 2008). As any newborn neurons that preferentially incorporated into existing neural circuitry could affect spatial memory (Kee et al, 2007) and that recalled of learned task 

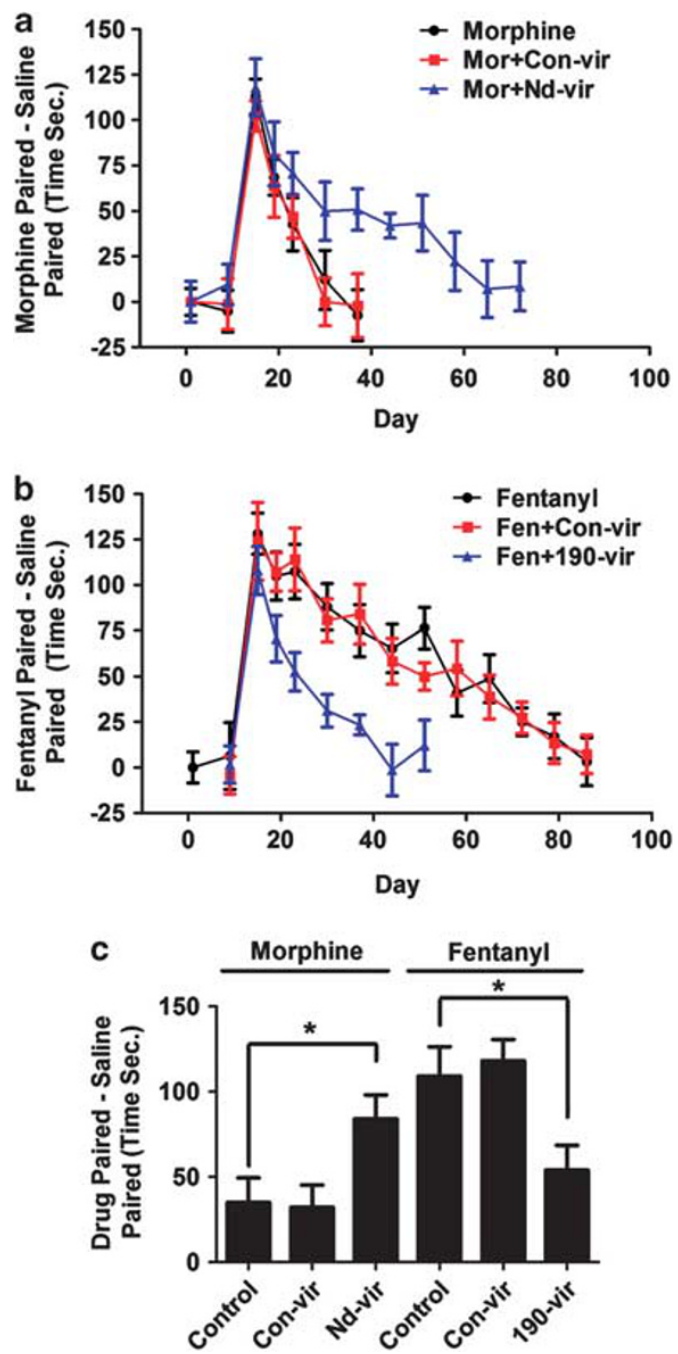

Figure 4 Neurogenic differentiation I (NeuroD) activity regulates conditioned place preference (CPP) extinction. Seven groups of mice were trained as in Figure $2 \mathrm{a}$ and $\mathrm{b}$. The acquisition of place preference was determined in the seven groups of mice. The extinction of place preference was tested every 7 days until no significant place preference was detected in two continuous tests. (c) After the extinction session, mice were challenged with morphine or fentanyl I week after the last place preference test. Recovered place preference was used to determine the reinstatement of CPP-related contextual memory. One-way ANOVA with Dunnett's test as a post-hoc test was used for analysis. Comparisons were done as indicated. Error bars and '*' represent SD $(n=10)$ and $P<0.0$ I, respectively.

involves the activation of adult-generated granular cells (Trouche et al, 2009), it is reasonable to hypothesize that by regulating adult neurogenesis, NeuroD contributes to the DG neural circuitry thereby regulating drug experience. This is exactly our observation. By manipulating NeuroD activity, either by drug administration or injection of lentivirus, the retention of contextual memory or drugassociated contextual memory can be modified. As CPP involves Pavlovian conditioning and is a model for cueelicited drug taking, modulation of NeuroD activity resulting in changes in adult neurogenesis affects the reappearance of the behavior in a drug-associated context, ie, during reinstatement or relapse. Together with recently reported studies in which cocaine response can be altered by ablating adult neurogenesis (Noonan et al, 2010), our studies clearly indicate that reinstatement of CPP is closely related to adult neurogenesis. Whether NeuroD activity can also affect the primary rewarding properties of opioid drugs remains to be evaluated.

The entire process of neurogenesis (from neural progenitor cells to mature neurons) requires at least 1 month. In our current study, there are 2 weeks between virus injection and memory extinction experiment. Thus whether the drugmediated changes in neurogenesis could account for the observed behavioral changes is debatable. However, previous studies support our current observations correlating neurogenesis and behavioral changes. Firstly, the time between the disappearance of DCX expression and the acquisition of NeuN expression can be as short as 1 week (Snyder et al, 2009). Thus, 2 weeks after the disruption of NeuroD activity or DCX expression, the incorporation of newly matured neurons into memory networks can be affected. Secondly, the immature state of new neurons also influences animal behavior. As ablation of 4-28 day old neurons impaired memory long-term memory (Snyder et al, 2005), it is possible that opioid agonist treatment could alter the maturation and differentiation of these immature neurons in addition to the reported effect on the progenitor cells within the SGZ. The experience within the immature stage of new neurons affects later neural representation of the experience in the DG (Tashiro et al, 2007). Determination of Arc expression in BrdU + cells suggests that the recruitment of new neurons into spatial memory networks occurs as early as 2 weeks in mice (Kee et al, 2007). Thirdly, young immature granule neurons can be more active than mature neurons in the DG, and can mediate pattern separation or memory resolution involving novel events on top of existing memory (Aimone et al, 2011; Nakashiba et al, 2012). Thus, the extinction of CPP or MWM memory could be influenced by altered neurogenesis under the current paradigm.

Although the difference in adult neurogenesis may lead to the different memory extinction, the other possibilities still exist. For example, chronic treatment with morphine but not fentanyl decreased the stability of dendritic spines of the primary hippocampal neurons (Zheng et al, 2010a). Thus the decreased spine stability by chronic morphine may also contribute to the reduced ability to maintain contextual memory under current paradigm. However, the main point of the current study was to indicate the correlations between the amounts of pNeuroD, adult neurogenesis in DG, and the extinction rate of contextual memory after different agonist treatment. The detailed mechanism on the drugmediated regulation of contextual memory should be investigated in future.

In summary, the transcription factor NeuroD represents an integration point of the opioid drug experience. Via differential control of miR-190 levels, morphine and fentanyl exhibit differential regulation of NeuroD activity, thereby resulting in differential modulation of adult neurogenesis and the extinction/reinstatement of the CPP response. Extending the reinstatement of CPP response to drug relapse, one could suggest that opioid drugs such as fentanyl that do not inhibit NeuroD activity will have a higher probability of inducing relapse than drugs such as morphine that inhibit NeuroD activity. If one could further suppress NeuroD activity, the extinction of the morphine 

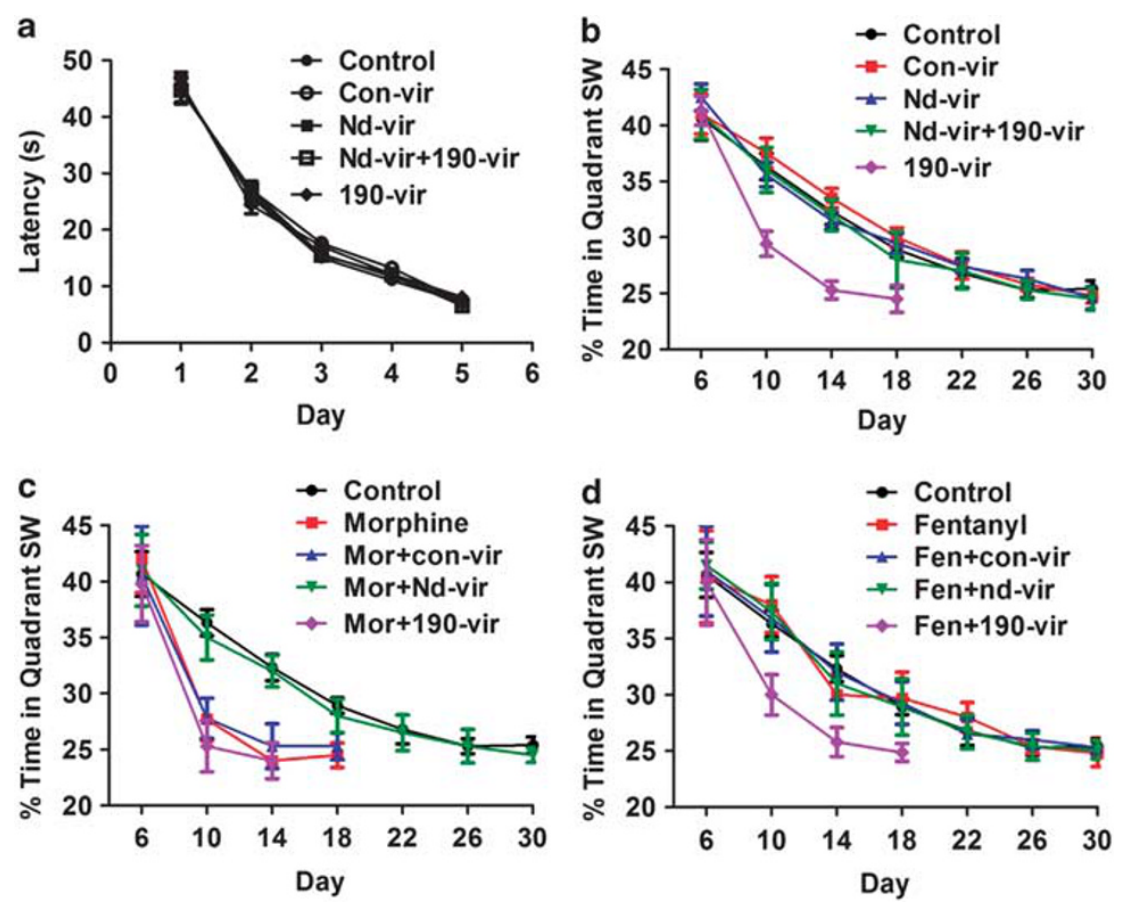

Figure 5 Neurogenic differentiation I (NeuroD) activity regulates Morris Water Maze (MWM)-related contextual memory. Control mice received no virus or agonist injection. Four groups of mice were injected with control oligonucleotide (Con-vir), NeuroD-lentivirus (Nd-vir), I90-virus (190-vir) + nd-vir (both 190-vir and nd-vir) or 190-vir (190-vir), respectively, but did not receive agonist injection. The other eight groups of mice (Morphine, Mor + con-vir, Mor + 190-vir, Mor + nd-vir, Fentanyl, Fen + con-vir, Fen + 190-vir, Fen + nd-vir) received both virus/vehicle injection and agonist injection before MWM training as in Supplementary Figure S4. (a) Latencies of mice in five groups (Control, Con-vir, Nd-vir, 190-vir + nd-vir and 190-vir) in finding the platform during the 5-day training. (b) A summary of the probe results of the five groups of mice in (a). The first day of MWM training was counted as day I and the training lasted from day I to day 5. After training, a probe test was used to determine remaining MWM-related contextual memory every 4 days until no significant memory was detected in two continuous tests. ( $c$ and d) A summary of the probe test results of the 'Control' group vs the other eight groups of mice (Morphine, Mor + con-vir, Mor + 190-vir, Mor + nd-vir, Fentanyl, Fen + con-vir, Fen + 190-vir, Fen + nd-vir). Error bars represent SD ( $n=10)$.

experience could be shortened further. Of note, injection of 190-vir before morphine training did shorten the CPP extinction time, but the difference was not statistically significant (Figure 5c). However, because NeuroD has numerous targets (Seo et al, 2007), whether it is possible to develop a paradigm in which NeuroD is targeted for drug relapse treatment might be difficult to ascertain.

\section{ACKNOWLEDGEMENTS}

This research was supported by the National Natural Science Foundation of China (31100773), the 'Guangzhou International Science and Technology Cooperation Projects from Bureau of Science and Information Technology of Guangzhou Municipal Government (2012J5100007)', the 'Guangdong Natural Science Foundation (S2012010010087)', and the NIH grants DA031442, DA023905 and DA011806.

\section{DISCLOSURE}

The authors declare no conflict of interest.

\section{REFERENCES}

Abrous DN, Koehl M, Le Moal M (2005). Adult neurogenesis: from precursors to network and physiology. Physiol Rev 85: 523-569.
Aguilar MA, Rodriguez-Arias M, Minarro J (2009). Neurobiological mechanisms of the reinstatement of drug-conditioned place preference. Brain Res Rev 59: 253-277.

Aimone JB, Deng W, Gage FH (2011). Resolving new memories: a critical look at the dentate gyrus, adult neurogenesis, and pattern separation. Neuron 70: 589-596.

Brown JP, Couillard-Despres S, Cooper-Kuhn CM, Winkler J, Aigner L, Kuhn HG (2003). Transient expression of doublecortin during adult neurogenesis. J Comp Neurol 467: 1-10.

Cetin A, Komai S, Eliava M, Seeburg PH, Osten P (2006). Stereotaxic gene delivery in the rodent brain. Nat Protoc 1: 3166-3173.

Chao J, Nestler EJ (2004). Molecular neurobiology of drug addiction. Annu Rev Med 55: 113-132.

Clelland CD, Choi M, Romberg C, Clemenson GD Jr, Fragniere A, Tyers P et al (2009). A functional role for adult hippocampal neurogenesis in spatial pattern separation. Science 325: 210-213.

Couillard-Despres S, Winner B, Schaubeck S, Aigner R, Vroemen $\mathrm{M}$, Weidner $\mathrm{N}$ et al (2005). Doublecortin expression levels in adult brain reflect neurogenesis. Eur J Neurosci 21: 1-14.

Cunningham CL, Gremel CM, Groblewski PA (2006). Druginduced conditioned place prefrence and aversion in mice. Nat Protocols 1: 1662-1670.

Eisch AJ, Harburg GC (2006). Opiates, psychostimulants, and adult hippocampal neurogenesis: insights for addiction and stem cell biology. Hippocampus 16: 271-286.

Fan GH, Wang LZ, Qiu HC, Ma L, Pei G (1999). Inhibition of calcium/calmodulin-dependent protein kinase II in rat hippocampus attenuates morphine tolerance and dependence. Mol Pharmacol 56: 39-45.

Fischer SJ, Arguello AA, Charlton JJ, Fuller DC, Zachariou V, Eisch AJ (2008). Morphine blood levels, dependence, and regulation of 
hippocampal subgranular zone proliferation rely on administration paradigm. Neuroscience 151: 1217-1224.

Fuchs RA, Evans KA, Ledford CC, Parker MP, Case JM, Mehta RH et al (2005). The role of the dorsomedial prefrontal cortex, basolateral amygdala, and dorsal hippocampus in contextual reinstatement of cocaine seeking in rats. Neuropsychopharmacology 30: 296-309.

Gaudilliere B, Konishi Y, de la Iglesia N, Yao G, Bonni A (2004). A CaMKII-NeuroD signaling pathway specifies dendritic morphogenesis. Neuron 41: 229-241.

Hernandez-Rabaza V, Hontecillas-Prieto L, Velazquez-Sanchez C, Ferragud A, Perez-Villaba A, Arcusa A et al (2008). The hippocampal dentate gyrus is essential for generating contextual memories of fear and drug-induced reward. Neurobiol Learn Mem 90: 553-559.

Hodge RD, Kowalczyk TD, Wolf SA, Encinas JM, Rippey C, Enikolopov $\mathrm{G}$ et al (2008). Intermediate progenitors in adult hippocampal neurogenesis: Tbr2 expression and coordinate regulation of neuronal output. J Neurosci 28: 3707-3717.

Kamath SG, Chen N, Enkemann SA, Sanchez-Ramos J (2005). Transcriptional profile of NeuroD expression in a human fetal astroglial cell line. Gene Expr 12: 123-136.

Kee N, Teixeira CM, Wang AH, Frankland PW (2007). Preferential incorporation of adult-generated granule cells into spatial memory networks in the dentate gyrus. Nat Neurosci 10: 355-362.

Lansink CS, Goltstein PM, Lankelma JV, McNaughton BL, Pennartz CM (2009). Hippocampus leads ventral striatum in replay of place-reward information. PLoS Biol 7: e1000173.

Lee I, Kesner RP (2004). Differential contributions of dorsal hippocampal subregions to memory acquisition and retrieval in contextual fear-conditioning. Hippocampus 14: 301-310.

Lee JK, Cho JH, Hwang WS, Lee YD, Reu DS, Suh-Kim H (2000). Expression of neuroD/BETA2 in mitotic and postmitotic neuronal cells during the development of nervous system. Dev Dyn 217: 361-367.

Lee JL (2008). Memory reconsolidation mediates the strengthening of memories by additional learning. Nat Neurosci 11: 1264-1266.

Liu K, Liu Y, Mo W, Qiu R, Wang X, Wu JY et al (2010). MiR-124 regulates early neurogenesis in the optic vesicle and forebrain, targeting NeuroD1. Nucleic Acids Res 39: 2859-2879.

McCormick MB, Tamimi RM, Snider L, Asakura A, Bergstrom D, Tapscott SJ (1996). NeuroD2 and neuroD3: distinct expression patterns and transcriptional activation potentials within the neuroD gene family. Mol Cell Biol 16: 5792-5800.

Milekic MH, Brown SD, Castellini C, Alberini CM (2006). Persistent disruption of an established morphine conditioned place preference. J Neurosci 26: 3010-3020.

Nakashiba T, Cushman JD, Pelkey KA, Renaudineau S, Buhl DL, McHugh TJ et al (2012). Young dentate granule cells mediate pattern separation, whereas old granule cells facilitate pattern completion. Cell 149: 188-201.

Noonan MA, Bulin SE, Fuller DC, Eisch AJ (2010). Reduction of adult hippocampal neurogenesis confers vulnerability in an animal model of cocaine addiction. J Neurosci 30: 304-315.

Restivo L, Vetere G, Bontempi B, Ammassari-Teule M (2009). The formation of recent and remote memory is associated with timedependent formation of dendritic spines in the hippocampus and anterior cingulate cortex. J Neurosci 29: 8206-8214.

Robinson TE, Berridge KC (2003). Addiction. Annu Rev Psychol 54: 25-53.

Rothwell PE, Gewirtz JC, Thomas MJ (2010). Episodic withdrawal promotes psychomotor sensitization to morphine. Neuropsychopharmacology 35: 2579-2589.
Seo S, Lim JW, Yellajoshyula D, Chang LW, Kroll KL (2007). Neurogenin and NeuroD direct transcriptional targets and their regulatory enhancers. Embo J 26: 5093-5108.

Shaham Y, Shalev U, Lu L, De Wit H, Stewart J (2003). The reinstatement model of drug relapse: history, methodology and major findings. Psychopharmacol 150: 337-346.

Shalev U, Grimm JW, Shaham Y (2002). Neurobiology of relapse to heroin and cocaine seeking: a review. Pharmacol Rev 54: 1-42.

Singer AC, Frank LM (2009). Rewarded outcomes enhance reactivation of experience in the hippocampus. Neuron 64: 910-921.

Snyder JS, Choe JS, Clifford MA, Jeurling SI, Hurley P, Brown A et al (2009). Adult-born hippocampal neurons are more numerous, faster maturing, and more involved in behavior in rats than in mice. J Neurosci 29: 14484-14495.

Snyder JS, Hong NS, McDonald RJ, Wojtowicz JM (2005). A role for adult neurogenesis in spatial long-term memory. Neuroscience 130: 843-852.

Tashiro A, Makino H, Gage FH (2007). Experience-specific functional modification of the dentate gyrus through adult neurogenesis: a critical period during an immature stage. $J$ Neurosci 27: 3252-3259.

Thomas JL, Ochocinska MJ, Hitchcock PF, Thummel R (2012). Using the $\mathrm{Tg}(\mathrm{nrd}$ :egfp)/albino zebrafish line to characterize in vivo expression of neurod. PLoS One 7: e29128.

Trouche S, Bontempi B, Roullet P, Rampon C (2009). Recruitment of adult-generated neurons into functional hippocampal networks contributes to updating and strengthening of spatial memory. Proc Natl Acad Sci USA 106: 5919-5924.

Tzschentke TM (2007). Measuring reward with the conditioned place preference (CPP) paradigm: update of the last decade. Addict Biol 12: 227-462.

Uittenbogaard M, Chiaramello A (2000). Differential expression patterns of the basic helix-loop-helix transcription factors during aging of the murine brain. Neurosci Lett 280: 95-98.

von Bohlen Und Halbach O (2007). Immunohistological markers for staging neurogenesis in adult hippocampus. Cell Tissue Res 329: 409-420.

Vorhees CV MT, Williams MT (2006). Morris water maze: procedures for assessing spatial and related forms of learning and memory. Nat Protoc 1: 848-858.

Weed MR, Hienz RD (2006). Effects of morphine on circadian rhythms of motor activity and body temperature in pig-tailed macaques. Pharmacol Biochem Behav 84: 487-496.

Zhang ZW, Zhang LQ, Ding L, Wang F, Sun YJ, An Y et al (2011). MicroRNA-19b downregulates insulin1 through targeting transcription factor NeuroD1. FEBS Lett 585: 2592-2598.

Zhao C, Deng W, Gage FH (2008). Mechanisms and functional implications of adult neurogenesis. Cell 132: 645-660.

Zheng B, De Vries L, Gist Farquhar M (1999). Divergence of RGS proteins: evidence for the existence of six mammalian RGS subfamilies. Trends Biochem Sci 24: 411-414.

Zheng H, Loh HH, Law PY (2008). ß-Arrestin-dependent $\mu$-opioid receptor-activated extracellular signal-regulated kinases (ERKs) translocate to nucleus in contrast to G protein-dependent ERK activation. Mol Pharmacol 73: 178-190.

Zheng H, Zeng Y, Chu J, Kam AY, Loh HH, Law PY (2010a). Modulations of NeuroD activity contribute to the differential effects of morphine and fentanyl on dendritic spine stability. J Neurosci 30: 8102-8110.

Zheng H, Zeng Y, Zhang X, Chu J, Loh HH, Law PY (2010b). $\mu-$ Opioid receptor agonists differentially regulate the expression of miR-190 and NeuroD. Mol Pharmacol 77: 102-109. 